\title{
Are Toll-like receptor gene polymorphisms associated with prostate cancer?
}

\author{
This article was published in the following Dove Press journal: \\ Cancer Management and Research \\ 4 February 2012 \\ Number of times this article has been viewed
}

\author{
Anton G Kutikhin \\ Arseniy E Yuzhalin \\ Department of Epidemiology, \\ Kemerovo State Medical Academy, \\ Kemerovo, Russian Federation
}

Correspondence: Arseniy E Yuzhalin Kemerovo State Medical Academy, Voroshilova Street, 22a, Kemerovo 650029, Russian Federation $\mathrm{Tel}+79235251517$ (mobile), +7 3842 75। 744 (work) Fax +7 384225 I 744 Email yuzhalin@gmail.com
Abstract: The suggestion that there is a connection between chronic intraprostatic inflammation and prostate cancer was declared some years ago. As Toll-like receptors (TLRs) are the key players in the processes of chronic intraprostatic inflammation, there is a hypothesis that $T L R$ gene polymorphisms may be associated with prostate cancer risk. Although a number of comprehensive studies have been conducted on large samples in various countries, reliable connections between these single nucleotide polymorphisms and prostate cancer risk, stage, grade, aggressiveness, ability to metastasize, and mortality have not been detected. Results have also varied slightly in different populations. The data obtained regarding the absence of connection between the polymorphisms of the genes encoding interleukin-1 receptor-associated kinases (IRAK1 and IRAK4) and prostate cancer risk might indicate a lack of association between inherited variation in the TLR signaling pathway and prostate cancer risk. It is possible to consider that polymorphisms of genes encoding TLRs and proteins of the TLR pathway also do not play a major role in the etiology and pathogenesis of prostate cancer. Feasibly, it would be better to focus research on associations between TLR single nucleotide polymorphisms and cancer risk in other infection-related cancer types.

Keywords: TLRs, single nucleotide polymorphisms, genetic variation, inflammation, innate immunity

\section{Discussion}

The results of a number of studies investigating the connections between sexually transmitted infections and prostatitis, between prostatitis and prostate cancer, and between genetic and circulating markers of inflammation and response to infection all support the hypothesis that there is a connection between chronic intraprostatic inflammation and prostate cancer. ${ }^{1}$ The list of causes of such inflammation includes exposure to various infectious agents, autoimmune disorders, damage from mechanical injuries, and chemical carcinogens (as exogenous as endogenous, for instance, certain hormones). ${ }^{1}$

Toll-like receptors (TLRs) constitute a family of receptors that recognize pathogenassociated and damage-associated molecular patterns, consequently playing a key role in innate and adaptive immune response, initiating the aforementioned inflammation. It has been suggested that TLR gene polymorphisms may affect TLR signaling, and, as a consequence, may influence TLR-mediated immune response, modulating prostate cancer risk. $^{2}$

Since 2004, when Zheng et $\mathrm{al}^{2}$ published the first paper devoted to the investigation of the role of TLR single nucleotide polymorphisms (SNPs) in cancer etiology, a 
number of other studies on this subject have been carried out. Nevertheless, results are rather discouraging: although Zheng et $\mathrm{al}^{2}$ found the rs 11536889 polymorphism is associated with increased prostate cancer risk and Chen et $\mathrm{al}^{3}$ observed that the $\mathrm{G}$ allele of the rs2770150 polymorphism may be a highrisk one, Lindström et al's ${ }^{7}$ recent meta-analysis combining the results of Zheng et $\mathrm{al}^{2}$ and Chen et $\mathrm{al}^{3}$ with three more large comprehensive studies ${ }^{4-6}$ did not reveal any correlation between TLR gene polymorphisms and prostate cancer risk. In addition, no high-risk alleles were detected in a large study by Stevens et al, ${ }^{8}$ not included in Lindström et al's ${ }^{7}$ pooled analysis. The results of all four large studies ${ }^{4-6,8}$ devoted to the association of polymorphisms of the TLR6-1-10 gene cluster with cancer risk suggest there is no correlation and that these SNPs cannot be considered promising for the further analysis of their association with prostate cancer risk. Balistreri et $\mathrm{al}^{9}$ obtained similar null results for TLR2 and $T L R 4$ SNPs. Positive results were found only for TLR4 gene polymorphisms by Cheng et $\mathrm{al}^{10}$ (rs10759932, odds ratio $[\mathrm{OR}]=4.62,95 \%$ confidence interval $[\mathrm{CI}]: 1.55-13.78$ for variant homozygous genotype), Song et al ${ }^{11}$ (rs1927911, $\mathrm{OR}=2.73,95 \% \mathrm{CI}: 1.54-4.87$ for heterozygous genotype; $\mathrm{OR}=6.68,95 \% \mathrm{CI}: 3.27-13.66$ for variant homozygous genotype; rs $11536858, \mathrm{OR}=2.3,95 \% \mathrm{CI}: 1.07-4.93$ for heterozygous genotype), Wang et $\mathrm{al}^{12}$ (rs10116253, $\mathrm{OR}=3.05,95 \% \mathrm{CI}: 1.11-8.41$ for variant homozygous genotype), and $\mathrm{Kim}$ et $\mathrm{al}^{13}$ (rs11536889, OR $=1.81,95 \%$ CI: 1.29-2.53 for heterozygous genotype). However, these SNPs were not detected as risk factors in Lindström et al's ${ }^{7}$ meta-analysis and therefore it is not possible to consider them as definite risk factors overall. Additionally, Shui et al, ${ }^{14}$ who carried out the most recent large investigation on this subject, did not detect any association between TLR4 gene polymorphisms and prostate cancer risk. The results of all studies mentioned are summarized in Tables 1 and 2 .

All of those who have investigated the association between $T L R$ gene polymorphisms and features of prostate cancer pathogenesis (stage, aggressiveness, Gleason grade, metastases), as well as the association between TLR gene polymorphisms and prostate cancer mortality, obtained negative results. This suggests there is no connection between $T L R$ gene polymorphisms and the pathogenetic peculiarities of prostate cancer. ${ }^{2-4,6,7,11,13,14}$

The active investigation of a correlation between TLR SNPS and prostate cancer is intriguing. Despite there being some fundamental mechanisms that indicate TLR gene polymorphisms may play a role in prostate cancer etiology, and despite there being a number of comprehensive studies conducted
Table I Association of TLR2 and TLR4 gene polymorphisms with prostate cancer risk

\begin{tabular}{|c|c|c|c|}
\hline Reference & SNP & Sample size & OR $(95 \% \mathrm{Cl}) *$ \\
\hline \multicolumn{4}{|l|}{ TLR2 } \\
\hline $\begin{array}{l}\text { Balistreri } \\
\text { et al }{ }^{9} \\
\text { (Italian } \\
\text { population) }\end{array}$ & $\begin{array}{l}\mathrm{rs} 5743708 \\
2029 \mathrm{C} / \mathrm{T}\end{array}$ & $\begin{array}{l}50 \text { cases, } \\
125 \text { controls, } \\
55 \text { male } \\
\text { centenarians }\end{array}$ & $\begin{array}{l}\text { NA } \\
\text { NA } \\
\text { (with age-matched } \\
\text { controls) }\end{array}$ \\
\hline \multicolumn{4}{|l|}{ TLR4 } \\
\hline \multirow[t]{4}{*}{$\begin{array}{l}\text { Zheng et al } \\
\text { (Swedish }^{2} \\
\text { population) }\end{array}$} & rsII536889 & $\begin{array}{l}\text { I } 383 \text { cases, } \\
780 \text { controls }\end{array}$ & $\begin{array}{l}\text { Carriers of } \\
\text { C allele: } 1.26 \\
\text { (I.0I-I.57) } \\
\text { [Before } 65 \text { years: } \\
\text { I.39 }(1.02-1.91)]\end{array}$ \\
\hline & rs503072I & & NA \\
\hline & rs4986790 & & NA \\
\hline & rs2149356 & & NA \\
\hline \multirow[t]{16}{*}{$\begin{array}{l}\text { Chen } \\
{\text { et } a l^{3} \text { (US }}_{\text {population) }}\end{array}$} & rs2770I50 & $\begin{array}{l}700 \text { cases, } \\
700 \text { controls }\end{array}$ & $\begin{array}{l}\text { Carriers of one } \\
\text { G allele: } 1.38 \\
(1.10-1.73)\end{array}$ \\
\hline & rsII536858 & & NA \\
\hline & rs64783I7 & & $\begin{array}{l}\text { Carriers of GG } \\
\text { genotype: } 0.66 \\
(0.46-0.94)\end{array}$ \\
\hline & rs10116253 & & $\begin{array}{l}\text { Carriers of CC } \\
\text { genotype: } 0.59 \\
(0.39-0.90)\end{array}$ \\
\hline & rs 1927914 & & $\begin{array}{l}\text { Carriers of GG } \\
\text { genotype: } 0.64 \\
(0.45-0.93)\end{array}$ \\
\hline & rs10759932 & & $\begin{array}{l}\text { Carriers of one } \\
\text { C allele: } 0.73 \\
(0.57-0.93)\end{array}$ \\
\hline & rs19279|I & & $\begin{array}{l}\text { Carriers of AA } \\
\text { genotype: } 0.63 \\
(0.4 I-0.95)\end{array}$ \\
\hline & rsII536878 & & NA \\
\hline & rs50307I7 & & $\begin{array}{l}\text { Carriers of one } \\
\text { G allele: } 0.66 \\
(0.51-0.86)\end{array}$ \\
\hline & rs2149356 & & $\begin{array}{l}\text { Carriers of TT } \\
\text { genotype: } 0.64 \\
(0.45-0.91)\end{array}$ \\
\hline & rs4986790 & & NA \\
\hline & rsII536889 & & NA \\
\hline & rs7873784 & & $\begin{array}{l}\text { Carriers of CC } \\
\text { genotype: } 0.5 \text { I } \\
(0.28-0.96)\end{array}$ \\
\hline & rsII53689| & & $\begin{array}{l}\text { Carriers of CC } \\
\text { genotype: } 0.50 \\
(0.27-0.95)\end{array}$ \\
\hline & rsII536897 & & NA \\
\hline & rs 1536898 & & $\begin{array}{l}\text { Carriers of AA } \\
\text { genotype: } 0.38 \\
(0.16-0.92)\end{array}$ \\
\hline $\begin{array}{l}\text { Cheng } \\
\text { et al }{ }^{10} \text { (US } \\
\text { population) }\end{array}$ & rs10759932 & $\begin{array}{l}506 \text { cases, } \\
506 \text { controls }\end{array}$ & $\begin{array}{l}\text { Carriers of CC } \\
\text { genotype: } 4.62 \\
(1.55-13.78)\end{array}$ \\
\hline & rs2149356 & & NA \\
\hline
\end{tabular}

(Continued) 
Table I (Continued)

\begin{tabular}{|c|c|c|c|c|c|c|c|}
\hline Reference & SNP & Sample size & OR $(95 \% \mathrm{CI}) *$ & Reference & SNP & Sample size & OR $(95 \% \mathrm{Cl}) *$ \\
\hline & rs5030728 & & Carriers of AA & & rs 10116253 & & Carriers of CC \\
\hline & & & genotype: 0.91 & & & & genotype: 3.05 \\
\hline & & & $(0.70-1.19)$ & & & & $(I . I I-8.4 I)$ \\
\hline & rs4986790 & & NA & & rs19279II & & NA \\
\hline & rsll1536889 & & NA & & rs|9279|4 & & NA \\
\hline & rs7873784 & & NA & & rs2149356 & & NA \\
\hline \multirow{26}{*}{$\begin{array}{l}\text { Yeager et al } \\
\text { (European } \\
\text { population) }\end{array}$} & rs1928298 & II 72 cases, & NA & & rs7873784 & & NA \\
\hline & & II 57 controls & & & rs|l53689| & & NA \\
\hline & & & & & rsII536898 & & NA \\
\hline & rsI360094 & & NA & & rs 2737190 & & NA \\
\hline & rs4837496 & & NA & Balistreri & rs4986790 & 50 cases, 125 age- & NA \\
\hline & rs 10818070 & & NA & et $\mathrm{al}^{9}$ & & matched controls, & \\
\hline & rs 10759930 & & NA & (Italian & & 55 centenarian & \\
\hline & rs2737191 & & NA & population) & & controls & \\
\hline & rs 2770150 & & NA & & rs498679l & & NA (with \\
\hline & rs6478317 & & NA & & & & age-matched \\
\hline & rsI0II6253 & & NA & & & & controls) \\
\hline & rs19279|4 & & NA & Lindström & rs|928298 & Pooled analysis: & NA \\
\hline & rs10759932 & & NA & et $\mathrm{al}^{7}$ & & 3101 cases, & \\
\hline & rs|9279|I & & NA & (meta-analysis & & 2253 controls & \\
\hline & rsII536879 & & NA & of Zheng & & & \\
\hline & rs50307I7 & & NA & et $\mathrm{al}^{2}{ }^{2}$ & & & \\
\hline & rs2149356 & & NA & Chen et $\mathrm{al}^{3}{ }^{3}$ & & & \\
\hline & rs4986790 & & NA & and Yeager & & & \\
\hline & rs7873784 & & NA & et $\left.\mathrm{al}^{5}\right)$ & & & \\
\hline & rsII536897 & & NA & & rs|360094 & & NA \\
\hline & rs1927906 & & NA & & rs4837496 & & NA \\
\hline & rsII536898 & & NA & & rs 10818070 & & NA \\
\hline & rsI554973 & & NA & & rs 10759930 & & NA \\
\hline & rs913930 & & NA & & rs2737|9| & & NA \\
\hline & rs1927905 & & NA & & rs 2770150 & & NA \\
\hline & rs7045953 & & NA & & rsII536858 & & NA \\
\hline \multirow{12}{*}{$\begin{array}{l}\text { Song et al" } \\
\text { (Korean } \\
\text { population) }\end{array}$} & rs|9279|I & 157 cases, & Carriers of TC & & rs6478317 & & NA \\
\hline & & I43 controls & genotype: 2.73 & & rs10116253 & & NA \\
\hline & & & $(1.54-4.87)$ & & rs 1927914 & & NA \\
\hline & & & Carriers of $\mathrm{CC}$ & & rs 10759932 & & NA \\
\hline & & & genotype: 6.68 & & rs 192791I & & NA \\
\hline & & & $(3.27-13.66)$ & & rs 10759933 & & NA \\
\hline & rsII536858 & & Carriers of GG & & rsII53687I & & NA \\
\hline & & & genotype: 2.3 & & rsII536879 & & NA \\
\hline & & & $(1.07-4.93)$ & & rs50303I7 & & NA \\
\hline & rs19279|4 & & NA & & rs2149356 & & NA \\
\hline & rsII53689I & & NA & & rs4986790 & & NA \\
\hline & rsII536897 & & NA & & rs503072I & & NA \\
\hline \multirow{14}{*}{$\begin{array}{l}\text { Wang } \\
\text { et al' }{ }^{12} \text { (US } \\
\text { population) }\end{array}$} & rs4986790 & 258 cases, & Carriers of $G$ & & rsII536889 & & NA \\
\hline & & 258 controls & allele: 0.60 & & rs7873784 & & NA \\
\hline & & & $(0.33-1.08)$ & & rsl|53689I & & NA \\
\hline & & & [Men younger & & rsII536897 & & NA \\
\hline & & & than 65 years: 0.26 & & rs 1927906 & & NA \\
\hline & & & $(0.08-0.87)]$ & & rsll536898 & & NA \\
\hline & rsII536889 & & Carriers of $\mathrm{C}$ & & rsI554973 & & NA \\
\hline & & & allele: 0.50 & & rs913930 & & NA \\
\hline & & & $(0.28-0.89)$ & & rs1927905 & & NA \\
\hline & & & (patients with normal & & rs7045953 & & NA \\
\hline & & & cholesterol) & Kim et $\mathrm{al}^{13}$ & rs 10983755 & 240 cases, & NA \\
\hline & & & $1.65(0.98-2.78)$ & (Korean & & 223 controls & \\
\hline & & & (patients with & population) & & & \\
\hline & & & elevated cholesterol) & & rs 10759932 & & NA \\
\hline
\end{tabular}

Table I (Continued)

NA (with 
Table I (Continued)

\begin{tabular}{|c|c|c|c|}
\hline Reference & SNP & Sample size & OR $(95 \% \mathrm{Cl}) *$ \\
\hline & rs19279II & & NA \\
\hline & rsII536879 & & NA \\
\hline & rs 12377632 & & NA \\
\hline & rs50307I7 & & NA \\
\hline & rs2149356 & & NA \\
\hline & rs50307I8 & & NA \\
\hline & rs7869402 & & NA \\
\hline & rsII536889 & & 1.81 (1.29-2.53) \\
\hline & & & (for heterozygous \\
\hline & & & genotype) \\
\hline & rs7873784 & & NA \\
\hline \multirow{11}{*}{$\begin{array}{l}\text { Shui } \\
\text { et al }{ }^{14} \text { (US } \\
\text { population) }\end{array}$} & Ten $T L R 4$ gene & I 286 cases, & NA \\
\hline & polymorphisms & 1267 controls & \\
\hline & & & NA \\
\hline & & & NA \\
\hline & & & NA \\
\hline & & & NA \\
\hline & & & NA \\
\hline & & & NA \\
\hline & & & NA \\
\hline & & & NA \\
\hline & & & NA \\
\hline
\end{tabular}

Note: *Only positive or negative statistically significant results.

Abbreviations: $\mathrm{Cl}$, confidence interval; NA, no association; OR, odds ratio; SNP, single nucleotide polymorphism; TLR, Toll-like receptor; US, United States.

on large samples in various countries, reliable connections between these SNPs and prostate cancer risk or features of prostate cancer progression have not been detected. Results have also varied slightly in different populations. However, it is possible that some of the TLR gene polymorphisms may be the markers of prostate cancer risk in certain populations (eg, rs5743795, rs5743551, rs5743556, rs5743604, rs4274855, rs11096957, rs11096955, and rs4129009 in the Swedish population; ${ }^{4}$ rs11536889 in the Swedish and the Korean populations; ${ }^{2,13}$ rs2770150, s10759932, and rs10116253 in the US population; ${ }^{3,10,12}$ rs 1927911 and rs11536858 in the Korean population $^{11}$ ). However, Lindström et al's ${ }^{7}$ meta-analysis, in which all of the populations mentioned above were considered, revealed that TLR gene polymorphisms cannot be the markers of prostate cancer overall and therefore they should be considered as risk markers, even in populations where the association has been found. ${ }^{7}$ Apparently, the lack of sample size was not the reason for negative results in either the general meta-analysis or in specific studies in particular populations, because the investigations in Swedish, ${ }^{2,4,15}$ European, ${ }^{5}$ and US populations $3,6,8,10,12$ included a large number of case and control subjects. Although two Korean studies ${ }^{11,13}$ had relatively small sample sizes, a recent large study in the Korean population also
Table 2 Association of polymorphisms of TLR6- I- 10 gene cluster with prostate cancer risk

\begin{tabular}{|c|c|c|c|}
\hline Reference & SNP & Sample size & OR $(95 \% \mathrm{Cl}) *$ \\
\hline \multirow{55}{*}{$\begin{array}{l}\text { Stevens } \\
\text { et al }{ }^{8} \text { (US } \\
\text { population) }\end{array}$} & TLRI0: & 1414 cases, & NA \\
\hline & rs4129009 & I4I4 controls & \\
\hline & (MAF I8\%-I8.5\%) & & \\
\hline & rs|l466657 & & NA \\
\hline & (MAF 3.09\%-3.38\%) & & \\
\hline & rs II466655 & & NA \\
\hline & (MAF $0.72 \%-0.76 \%)$ & & \\
\hline & rsII096955 & & A/C compared \\
\hline & (MAF 32.6\%-35.8\%) & & with $A / A: 0.84$ \\
\hline & & & $(0.72-0.98)$ \\
\hline & & & $\mathrm{C} / \mathrm{C}$ compared \\
\hline & & & with $A / A: 0.78$ \\
\hline & & & $(0.6 \mathrm{I}-0.99)$ \\
\hline & rsII096956 & & NA \\
\hline & (MAF $21.1 \%-23.5 \%)$ & & \\
\hline & rs|l466653 & & NA \\
\hline & (MAF 2.94\%-3.93\%) & & \\
\hline & rsI|46665I & & NA \\
\hline & (MAF $3.14 \%-3.74 \%)$ & & \\
\hline & rsII096957 & & $\mathrm{A} / \mathrm{C}$ compared \\
\hline & (MAF 32.6\%-35.8\%) & & with $A / A: 0.84$ \\
\hline & & & $(0.72-0.98)$ \\
\hline & & & $\mathrm{C} / \mathrm{C}$ compared \\
\hline & & & with $A / A: 0.78$ \\
\hline & & & $(0.6 \mathrm{I}-0.99)$ \\
\hline & rs II466649 & & NA \\
\hline & (MAF 3.3\%-3.84\%) & & \\
\hline & rs 10856838 & & NA \\
\hline & (MAF I4.7\%-16.4\%) & & \\
\hline & rs4274855 & & NA \\
\hline & (MAF I8\%-18.5\%) & & \\
\hline & rs|l466640 & & NA \\
\hline & (MAF I8.1\%-18.6\%) & & \\
\hline & rsII466617 & & NA \\
\hline & (MAF I8\%-I8.6\%) & & \\
\hline & rs7653908 & & NA \\
\hline & (MAF 2I.I\%-20.6\%) & & \\
\hline & rs7658893 & & NA \\
\hline & (MAF 23.6\%-25.2\%) & & \\
\hline & TLRI: & & NA \\
\hline & rs4624663 & & \\
\hline & (MAF $2.46 \%-2.18 \%)$ & & \\
\hline & rs4833095 & & T/C compared \\
\hline & (MAF 23.4\%-26.8\%) & & with T/T: 0.90 \\
\hline & & & $(0.77-1.05)$ \\
\hline & & & C/C compared \\
\hline & & & with TT/T: 0.64 \\
\hline & & & $(0.47-0.86)$ \\
\hline & rs57436II & & NA \\
\hline & (MAF 8.6\%-8.8\%) & & \\
\hline & rs5743604 & & NA \\
\hline & (MAF 23.9\%-24.2\%) & & \\
\hline & rs57443596 & & $\mathrm{C} / \mathrm{T}$ compared \\
\hline & (MAF I4.9\%-I8.5\%) & & with C/C: 0.79 \\
\hline & & & $(0.66-0.93)$ \\
\hline
\end{tabular}


Table 2 (Continued)

\begin{tabular}{|c|c|c|c|}
\hline Reference & SNP & Sample size & OR $(95 \% \mathrm{Cl}) *$ \\
\hline & & & $\mathrm{T} / \mathrm{T}$ compared \\
\hline & & & with C/C: 0.59 \\
\hline & & & $(0.38-0.91)$ \\
\hline & rs5743595 & & T/C compared \\
\hline & (MAF I7.4\%-20.6\%) & & with T/T: 0.82 \\
\hline & & & $(0.70-0.97)$ \\
\hline & & & $\mathrm{C} / \mathrm{C}$ compared \\
\hline & & & with T/T: 0.63 \\
\hline & & & $(0.42-0.93)$ \\
\hline & rs5743594 & & NA \\
\hline & (MAF I9.8\%-I7.7\%) & & \\
\hline & rs5743556 & & NA \\
\hline & (MAF I9\%-19.6\%) & & \\
\hline & rs574355 I & & A/G compared \\
\hline & (MAF $23.7 \%-26.7 \%)$ & & with $A / A: 0.90$ \\
\hline & & & $(0.77-1.06)$ \\
\hline & & & G/G compared \\
\hline & & & with $A / A: 0.67$ \\
\hline & & & $(0.50-0.91)$ \\
\hline & TLR6: & & NA \\
\hline & rs57438I5 & & \\
\hline & (MAF I.9I\%-I.27\%) & & \\
\hline & rs5743810 & & NA \\
\hline & (MAF $42.1 \%-42.7 \%)$ & & \\
\hline & rs5743806 & & NA \\
\hline & (MAF $30.3 \%-30.6 \%)$ & & \\
\hline & rs5743795 & & NA \\
\hline & (MAF I9.9\%-20.2\%) & & \\
\hline \multirow{28}{*}{$\begin{array}{l}\text { Chen } \\
\text { et al }{ }^{6} \text { (US } \\
\text { population) }\end{array}$} & rs5743788 & 659 cases, & NA \\
\hline & (MAF 50\%-49\%) & 656 controls & \\
\hline & rs5743795 & & NA \\
\hline & (MAF I9\%-2I\%) & & \\
\hline & rs5743806 & & NA \\
\hline & (MAF 3I\%-30\%) & & \\
\hline & rs1039599 & & NA \\
\hline & (MAF 46\%-46\%) & & \\
\hline & rs5743810 & & NA \\
\hline & (MAF $42 \%-41 \%)$ & & \\
\hline & rs3821985 & & NA \\
\hline & (MAF 34\%-33\%) & & \\
\hline & rs57438I5 & & NA \\
\hline & (MAF I\%-2\%) & & \\
\hline & rs574355I & & NA \\
\hline & (MAF 24\%-26\%) & & \\
\hline & rs5743556 & & NA \\
\hline & (MAF I8\%-19\%) & & \\
\hline & rs57433604 & & NA \\
\hline & (MAF 23\%-26\%) & & \\
\hline & rs57436II & & NA \\
\hline & (MAF 8\%-9\%) & & \\
\hline & rs 4624663 & & NA \\
\hline & (MAF 4\%-4\%) & & \\
\hline & rsll 466617 & & NA \\
\hline & (MAF I7\%-I8\%) & & \\
\hline & rsII466640 & & NA \\
\hline & (MAF I7\%-19\%) & & \\
\hline
\end{tabular}

(Continued)
Table 2 (Continued)

\begin{tabular}{|c|c|c|c|}
\hline Reference & SNP & Sample size & OR $(95 \% \mathrm{Cl}) *$ \\
\hline & rs4274855 & & NA \\
\hline & (MAF I8\%-I9\%) & & \\
\hline & rsll096957 & & NA \\
\hline & (MAF 33\%-36\%) & & \\
\hline & rsll096955 & & NA \\
\hline & (MAF 33\%-36\%) & & \\
\hline & rsII466657 & & NA \\
\hline & MAF (4\%-4\%) & & \\
\hline & rs4129009 & & NA \\
\hline & (MAF I7\%-I8\%) & & \\
\hline \multirow{21}{*}{$\begin{array}{l}\text { Yeager et al }{ }^{5} \\
\text { (European } \\
\text { population) }\end{array}$} & rs 10008492 & II 72 cases, & NA \\
\hline & & II 57 controls & \\
\hline & & & \\
\hline & rs433I786 & & NA \\
\hline & rsII466657 & & NA \\
\hline & rsII096957 & & NA \\
\hline & rs 10856839 & & NA \\
\hline & rsII466640 & & NA \\
\hline & rsII466619 & & NA \\
\hline & rsII466612 & & NA \\
\hline & rs7663239 & & NA \\
\hline & rs 4543123 & & NA \\
\hline & rs4833095 & & NA \\
\hline & rs5743594 & & NA \\
\hline & rs5743563 & & NA \\
\hline & rs4833103 & & NA \\
\hline & rs7696175 & & NA \\
\hline & rs5743810 & & NA \\
\hline & rs1039559 & & NA \\
\hline & rs68339|4 & & NA \\
\hline & rs6531673 & & NA \\
\hline \multirow{26}{*}{$\begin{array}{l}\text { Sun et al }{ }^{4} \\
\text { (Swedish } \\
\text { population) }\end{array}$} & TLR6: & I383 cases, & NA \\
\hline & $2113 \mathrm{C} / \mathrm{G}$ & 780 controls & \\
\hline & (73.76\%-76.35\% & & \\
\hline & $\mathrm{C} / \mathrm{G}$ and $\mathrm{G} / \mathrm{G})$ & & \\
\hline & rs5743795 & & $\mathrm{A} / \mathrm{G}$ and $\mathrm{A} / \mathrm{A}$ \\
\hline & $(32.8 \%-26.24 \%$ & & compared with \\
\hline & $\mathrm{A} / \mathrm{G}$ and $\mathrm{AA})$ & & G/G: 1.38 \\
\hline & & & $(1.12-1.70)$ \\
\hline & rs5743806 & & $\mathrm{C} / \mathrm{T}$ and $\mathrm{T} / \mathrm{T}$ \\
\hline & (89.12\%-89.33\% & & compared with \\
\hline & $\mathrm{C} / \mathrm{T}$ and $\mathrm{T} / \mathrm{T})$ & & C/C: 0.98 \\
\hline & & & $(0.73-1.3 I)$ \\
\hline & rs5743810 & & NA \\
\hline & (82.84\%-82.84\% & & \\
\hline & $\mathrm{C} / \mathrm{T}$ and $\mathrm{C} / \mathrm{C}$ ) & & \\
\hline & rs57438I5 & & NA \\
\hline & $(3.44 \%-2.9 \%$ & & \\
\hline & $\mathrm{C} / \mathrm{T}$ and $\mathrm{C} / \mathrm{C}$ ) & & \\
\hline & TLRI: & & $A / G$ and $G / G$ \\
\hline & rs574355I & & compared \\
\hline & $(40.16 \%-34.32 \%$ & & with A/A: I.29 \\
\hline & $\mathrm{A} / \mathrm{G}$ and $\mathrm{G} / \mathrm{G})$ & & $(1.06-1.56)$ \\
\hline & rs5743556 & & $\mathrm{C} / \mathrm{T}$ and $\mathrm{C} / \mathrm{C}$ \\
\hline & (32.67\%-26.65\% & & compared with \\
\hline & $\mathrm{C} / \mathrm{T}$ and $\mathrm{C} / \mathrm{C}$ ) & & T/T: 1.33 \\
\hline & & & $(1.09-1.62)$ \\
\hline
\end{tabular}


Table 2 (Continued)

\begin{tabular}{|c|c|c|c|}
\hline Reference & SNP & Sample size & OR $(95 \% \mathrm{Cl}) *$ \\
\hline & rs5743604 & & $\mathrm{C} / \mathrm{T}$ and $\mathrm{C} / \mathrm{C}$ \\
\hline & $(42.1 \%-35.69 \%$ & & compared with \\
\hline & $\mathrm{C} / \mathrm{T}$ and $\mathrm{C} / \mathrm{C})$ & & $\mathrm{T} / \mathrm{T}: 1.30$ \\
\hline & & & $(1.08-1.60)$ \\
\hline & rs57436II & & NA \\
\hline & (98.35\%-98.21\% & & \\
\hline & $\mathrm{G} / \mathrm{C}$ and $\mathrm{G} / \mathrm{G})$ & & \\
\hline & rs 4624663 & & NA \\
\hline & (7.79\%-7.16\% & & \\
\hline & G/A and $G / G)$ & & \\
\hline & TLRIO: & & $\mathrm{T} / \mathrm{C}$ and $\mathrm{C} / \mathrm{C}$ \\
\hline & $3260 \mathrm{C} / \mathrm{T}$ & & compared with \\
\hline & $(29.79 \%-26.06 \%$ & & $\mathrm{T} / \mathrm{T}: \mathbf{I} .20$ \\
\hline & $\mathrm{T} / \mathrm{C}$ and $\mathrm{C} / \mathrm{C}$ ) & & $(0.99-1.46)$ \\
\hline & $\mathrm{I} 692 \mathrm{C} / \mathrm{T}$ & & $\mathrm{C} / \mathrm{T}$ and $\mathrm{T} / \mathrm{T}$ \\
\hline & $(30.34 \%-26.04 \%$ & & compared with \\
\hline & $\mathrm{C} / \mathrm{T}$ and $\mathrm{T} / \mathrm{T})$ & & C/C: 1.23 \\
\hline & & & $(1.0 \mathrm{I}-1.50)$ \\
\hline & rs4274855 & & $\mathrm{A} / \mathrm{G}$ and $\mathrm{A} / \mathrm{A}$ \\
\hline & (32.04\%-26.93\% & & compared with \\
\hline & $\mathrm{A} / \mathrm{G}$ and $\mathrm{A} / \mathrm{A})$ & & G/G: 1.27 \\
\hline & & & $(1.04-1.56)$ \\
\hline & rsII096957 & & $\mathrm{A} / \mathrm{C}$ and $\mathrm{C} / \mathrm{C}$ \\
\hline & $(60.18 \%-55.85 \%$ & & compared with \\
\hline & $\mathrm{A} / \mathrm{C}$ and $\mathrm{C} / \mathrm{C})$ & & $\mathrm{A} / \mathrm{A}: 1.20$ \\
\hline & & & $(1.00-1.43)$ \\
\hline & rsllo96955 & & $\mathrm{A} / \mathrm{C}$ and $\mathrm{C} / \mathrm{C}$ \\
\hline & (57.19\%-51.70\% & & compared with \\
\hline & $\mathrm{A} / \mathrm{C}$ and $\mathrm{C} / \mathrm{C})$ & & A/A: 1.25 \\
\hline & & & $(1.04-1.50)$ \\
\hline & rsII466657 & & NA \\
\hline & $(4.39 \%-4.08 \% \mathrm{~T} / \mathrm{C})$ & & \\
\hline & rs4l29009 & & $\mathrm{G} / \mathrm{A}$ and $\mathrm{G} / \mathrm{G}$ \\
\hline & (31.20\%-26.31\% & & compared with \\
\hline & $\mathrm{G} / \mathrm{A}$ and $\mathrm{G} / \mathrm{G})$ & & A/A: 1.26 \\
\hline & & & $(1.03-1.54)$ \\
\hline Lindström & rsl0008492 & $310 \mid$ cases, & NA \\
\hline et $\mathrm{al}^{7}$ & & 2523 controls & \\
\hline (meta-analysis & & & \\
\hline of Sun et $\mathrm{al}^{4}{ }^{4}$ & & & \\
\hline Chen et al, ${ }^{6}$ & & & \\
\hline and Yeager & & & \\
\hline et $\left.\left.a\right|^{5}\right)$ & & & \\
\hline & rs4331786 & & NA \\
\hline & rs4I29009 & & NA \\
\hline & rsI|4666657 & & NA \\
\hline & rsIl 096955 & & NA \\
\hline & rsII096957 & & NA \\
\hline & rs10856839 & & NA \\
\hline & rs4274855 & & NA \\
\hline & rsII466640 & & NA \\
\hline & rsII466619 & & NA \\
\hline & rsII466617 & & NA \\
\hline & rsII466612 & & NA \\
\hline & rs7663239 & & NA \\
\hline & rs 4543123 & & NA \\
\hline & rs4624663 & & NA \\
\hline
\end{tabular}

Table 2 (Continued)

\begin{tabular}{|c|c|c|c|}
\hline Reference & SNP & Sample size & OR $(95 \% \mathrm{Cl}) *$ \\
\hline & rs4833095 & & NA \\
\hline & rs57436II & & NA \\
\hline & rs5743604 & & NA \\
\hline & rs5743594 & & NA \\
\hline & rs5743563 & & NA \\
\hline & rs5743556 & & NA \\
\hline & rs574355I & & NA \\
\hline & rs4833I03 & & NA \\
\hline & rs7696I75 & & NA \\
\hline & rs57438I5 & & NA \\
\hline & rs3821985 & & NA \\
\hline & rs5743810 & & NA \\
\hline & rs1039559 & & NA \\
\hline & rs5743806 & & NA \\
\hline & rs5743795 & & NA \\
\hline & rs5743788 & & NA \\
\hline & rs68339|4 & & NA \\
\hline & rs6531673 & & NA \\
\hline
\end{tabular}

Note: *Only positive or negative statistically significant results.

Abbreviations: $\mathrm{Cl}$, confidence interval; MAF, minor allele frequency; OR, odds ratio; SNP, single nucleotide polymorphism; TLR, Toll-like receptor.

obtained negative results. ${ }^{14}$ Therefore, the statistical power of almost all of the studies was sufficient. Population stratification in various studies revealed no subcategorical differences when compared with general results, although a dependence of association on age was found in one study in the Swedish population, ${ }^{2}$ and cholesterol level was found to influence the association in one study in the US population. ${ }^{12}$ However, alone these results cannot provide sufficient information on the subcategorical modification of association of TLR gene polymorphisms with prostate cancer. In addition, there are no studies considering the gene-gene and gene-environment interactions in relation to prostate cancer.

Sun et $\mathrm{al}^{15}$ did not observe any correlation between polymorphisms of the genes encoding the interleukin-1 receptor-associated kinases (IRAK1 and IRAK4) and prostate cancer. The data obtained by Sun et $\mathrm{al}^{15}$ might also reflect a lack of association between inherited variation in genes encoding proteins of the TLR signaling pathway and prostate cancer risk, since IRAK1 and IRAK4 are key proteins of this pathway.

\section{Conclusion}

In conclusion, it is possible to suggest that TLR and TLR pathway gene polymorphisms do not play a major role in the etiology of prostate cancer, although in certain populations their minor role can be established. Feasibly, it would be better to focus research on associations between TLR SNPs and cancer risk in other infection-related cancer types. 


\section{Disclosure}

The authors report no conflicts of interest in this work.

\section{References}

1. De Marzo AM, Marchi VL, Epstein JI, Nelson WG. Proliferative inflammatory atrophy of the prostate: implications for prostatic carcinogenesis. Am J Pathol. 1999;155(6):1985-1992.

2. Zheng SL, Augustsson-Bälter K, Chang B, et al. Sequence variants of Toll-like receptor 4 are associated with prostate cancer risk: results from the CAncer Prostate in Sweden Study. Cancer Res. 2004;64(8):2918-2922.

3. Chen YC, Giovannucci E, Lazarus R, Kraft P, Ketkar S, Hunter DJ. Sequence variants of Toll-like receptor 4 and susceptibility to prostate cancer. Cancer Res. 2005;65(24):11771-11778.

4. Sun J, Wiklund F, Zheng SL, et al. Sequence variants in Toll-like receptor gene cluster (TLR6-TLR1-TLR10) and prostate cancer risk. $J$ Natl Cancer Inst. 2005;97(7):525-532.

5. Yeager M, Orr N, Hayes RB, et al. Genome-wide association study of prostate cancer identifies a second risk locus at 8q24. Nat Genet. 2007;39(5):645-649.

6. Chen YC, Giovannucci E, Kraft P, Lazarus R, Hunter DJ. Association between Toll-like receptor gene cluster (TLR6, TLR1, and TLR10) and prostate cancer. Cancer Epidemiol Biomarkers Prev. 2007;16(10):1982-1989.

7. Lindström S, Hunter DJ, Grönberg H, et al. Sequence variants in the TLR4 and TLR6-1-10 genes and prostate cancer risk: results based on pooled analysis from three independent studies. Cancer Epidemiol Biomarkers Prev. 2010;19(3):873-876.
8. Stevens VL, Hsing AW, Talbot JT, et al. Genetic variation in the Toll-like receptor gene cluster (TLR10-TLR1-TLR6) and prostate cancer risk. Int J Cancer 2008;123(11):2644-2650.

9. Balistreri CR, Caruso C, Carruba G, et al. A pilot study on prostate cancer risk and pro-inflammatory genotypes: pathophysiology and therapeutic implications. Curr Pharm Des. 2010;16(6):718-724.

10. Cheng I, Plummer SJ, Casey G, Witte JS. Toll-like receptor 4 genetic variation and advanced prostate cancer risk. Cancer Epidemiol Biomarkers Prev. 2007;16(2):352-355.

11. Song J, Kim DY, Kim CS, et al. The association between Toll-like receptor 4 (TLR4) polymorphisms and the risk of prostate cancer in Korean men. Cancer Genet Cytogenet. 2009;190(2):88-92.

12. Wang MH, Helzlsouer KJ, Smith MW, et al. Association of IL10 and other immune response- and obesity-related genes with prostate cancer in CLUE II. Prostate. 2009;69(8):874-885.

13. Kim HJ, Bae JS, Chang IH, et al. Sequence variants of Toll-like receptor 4 (TLR4) and the risk of prostate cancer in Korean men. World J Urol. Epub 2011 May 7.

14. Shui IM, Stark JR, Penney KL, et al. Genetic variation in the Toll-like receptor 4 and prostate cancer incidence and mortality. Prostate. Epub 2011 May 11.

15. Sun J, Wiklund F, Hsu FC, et al. Interactions of sequence variants in interleukin-1 receptor-associated kinase 4 and the Toll-like receptor 6-1-10 gene cluster increase prostate cancer risk. Cancer Epidemiol Biomarkers Prev. 2006;15(3):480-485.
Cancer Management and Research

\section{Publish your work in this journal}

Cancer Management and Research is an international, peer-reviewed open access journal focusing on cancer research and the optimal use of preventative and integrated treatment interventions to achieve improved outcomes, enhanced survival and quality of life for the cancer patient The journal welcomes original research, clinical \& epidemiological

\section{Dovepress}

studies, reviews \& evaluations, guidelines, expert opinion \& commentary, case reports \& extended reports. The manuscript management system is completely online and includes a very quick and fair peerreview system, which is all easy to use. Visit http://www.dovepress.com/ testimonials.php to read real quotes from published authors. 